\title{
Application of Novel Methods for Non-Small Cell Lung Cancer (NSCLC) Biomarker Discovery
}

\author{
Artjoms Spaks*,**, Ainis Pirtnieks*, Jelena Grusina-Ujumaza*,***, Jazeps Basko*, Dainis Krievins** \\ *Department of Thoracic Surgery, Pauls Stradins Clinical University Hospital, Riga, Latvia \\ **University of Latvia, Riga, Latvia \\ ***Riga Stradins University, Riga, Latvia
}

\begin{abstract}
Summary
Introduction. Research in NSCLC biomarker field and application of new methods is essential as it is promising strategy to reduce cancer mortality.

Aim of the study. The aim of the study was to develop new approach for lung cancer biomarker research and introduce novel method into surgical practice, verifying method's safety and evaluate intraoperative and postoperative complications.

Materials and methods. 50 patients with early stage NSCLC undergoing lobectomy were randomized into two groups - experimental $(n=25)$ and control group $(n=25)$. In experimental group at the time of thoracotomy and resection of the lung, paired blood samples were obtained from the lobar pulmonary vein draining lung segment containing tumor and from the peripheral vein which represents the systemic circulation. Safety of the procedure and its influence on surgery was evaluated.

Results. New approach for lung cancer biomarker research was developed. Injuries of major anatomical structures as pulmonary blood vessels, bronchi, trachea and pericardium due to blood sample collection were not observed. There was no difference in hospital stay and chest tube drainage duration. There were no perioperative deaths in both groups. Morbidity rate in experimental group was similar to control group.

Conclusions. Application of new method for developing multimodal lung cancer model is safe and does not increase the risk of intraoperative and postoperative complications.
\end{abstract}

Key words: biomarkers; lung cancer; lung resection.

\section{INTRODUCTION}

Lung cancer is the most common cancer worldwide with the number of incident cases around 1.6 million annually (14). Overall, survival rate at 5 years is less than $20 \%$ and the search for prognostic factors has led to extensive research and publication of an impressive number of papers. There are plenty of publications in the literature about lung cancer biological markers which can facilitate early detection of lung cancer in such a way improving results of treatment and improving 5-year survival. A blood based biomarkers are attractive targets because blood is easily accessible and measurements may be repeated over time $(5,9,16)$. Blood is a medium which carries information about cellular processes, tumor progression and growth, signaling and much more (20). It is enormous amount of data which cannot be processed without building strategy allowing targeted approach. Isolating blood draining tumor gives insight into metabolism of cancer cells. Combination of surgical practice with new research strategies could help to achieve progress in NSCLC biomarker discovery. We describe a novel approach to biomarker discovery that used the same subject as control to identify elevated proteins in the pulmonary venous effluent draining the tumor vascular bed compared to systemic blood. This approach allows the differentlially present proteins to be identified against a complex and variable background of proteomic profile. The analytic issue is reduced to determining what has changed in an individual pre- and post- passage through the affected lung to get around the problem of finding specific biomarkers in blood.

\section{AIM OF THE STUDY}

The aim of the study was to develop new approach for lung cancer biomarker research, introduce and incorporate novel method into surgical practice, verify method's safety and evaluate intraoperative and postoperative complications.

\section{MATERIAL AND METHODS}

This study was designed as a randomized, case-control study. Development of novel approach was incorporated in a study design of Pauls Stradins Clinical University Hospital Department of Thoracic Surgery scientific project "Investigation of CXC group chemokines - novel diagnostic biomarkers for early stage lung cancer".

Patients with early stage (IA - IIB, $7^{\text {th }}$ edition of TNM in Lung Cancer of the International Association for the Study of Lung Cancer) NSCLC undergoing lung resection with curative intent were involved in the study $(n=50)$ from January 2010 till December 2012. All patients participating in the study signed informed consent form.

A simple randomization was used. Patients were randomized into two groups - experimental $(\mathrm{n}=25)$ and control group $(n=25)$. In all patients extent of resection was radical and restricted to lobectomy. Open lobectomy and nodal dissection was performed 
in all patients. Steps of standard procedure were as follows - lateral thoracotomy, mobilization of lung, verification of tumor location, division of mediastinal and interlobar pleura, exposure and identification of hilar structures, dissection of pulmonary artery and vein, ligation and division of pulmonary vessels, dissection of lobar bronchus, division and closure of bronchus, haemostasis, drainage and wound closure. In experimental group at the time of thoracotomy and resection of the lung, paired blood samples were obtained from the lobar pulmonary vein draining lung segment containing tumor, and from the peripheral cubital vein which represents the systemic circulation. After dissection of pulmonary vein $5 \mathrm{~mL}$ of blood were aspirated with a syringe with 21 -gauge needle prior to blood vessel ligation (Fig. 1).

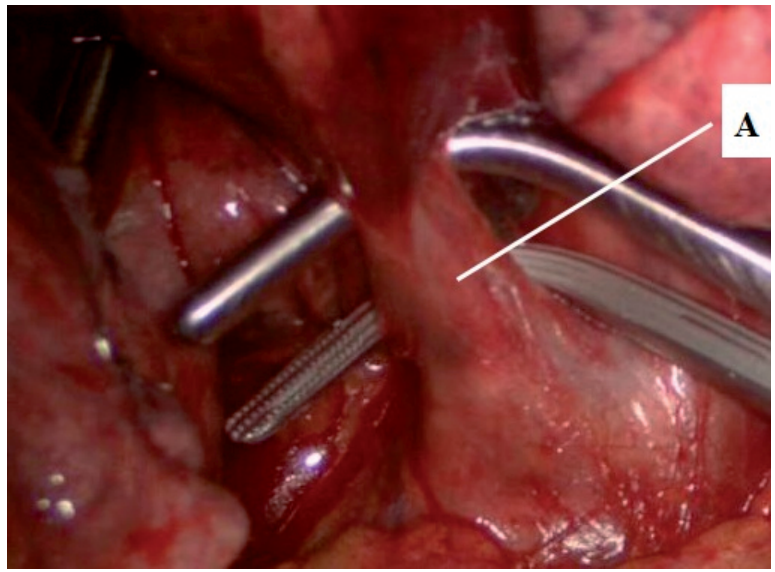

Fig. 1. Anterior view of dissected middle lobe vein of right lung before blood sample aspiration and vein ligation. Pulmonary vein puncture site (A) Afterwards pulmonary vein was ligated proximally to puncture site. Blood samples were processed during next two hours after collection - centrifuged at $3000 \mathrm{~g}$ for 10 minutes and stored at $-70^{\circ} \mathrm{C}$ for further analysis. Safety of procedure and its influence on surgery was evaluated. Various parameters were assessed including duration of surgery, intraoperative blood loss, postoperative hospital stay, duration of chest tube drainage, volume of postoperative fluid drained per day (postoperative day 1 to 3), haemoglobin concentration in blood and pleural fluid on the first postoperative day. Cardiac events were assessed intraoperatively and postoperatively - episodes of arrhythmia and miocardial ischemia were recorded. Data of two groups were compared.

Descriptive statistics and Student's paired t-test were used for the comparison of the means of numerical data.

\section{RESULTS}

Duration of surgery in experimental group was similar to control group $(148.8 \pm 37.1$ minutes versus $141 \pm 38$ minutes, $\mathrm{p}=0.47$ ). Intraoperative blood loss was slightly higher in experimental group, but difference was not statistically significant (482 $\pm 177 \mathrm{ml}$ (ranged $250-900$ $\mathrm{ml}$ ) versus $432 \pm 146$ (ranged $260-780 \mathrm{ml}$ ), $\mathrm{p}=0.28$ ). Volume of postoperative fluid drainage per day was similar in both groups with minor difference on the first day after surgery (Fig. 2).

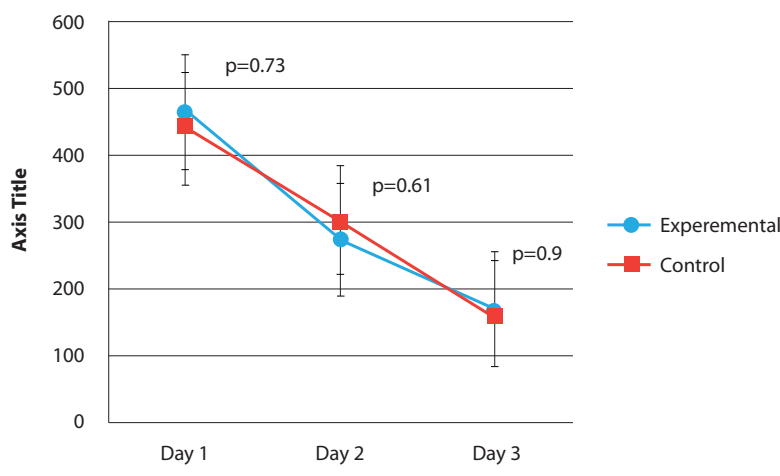

Fig. 2. Volume of postoperative fluid drainage per day (day $1-3)$. Values are expressed as the mean drainage (in milliliters) $\pm \mathrm{SE}$

Haemoglobin concentration in blood and pleural fluid on the first postoperative day was similar in experimental and control groups (Fig. 3).

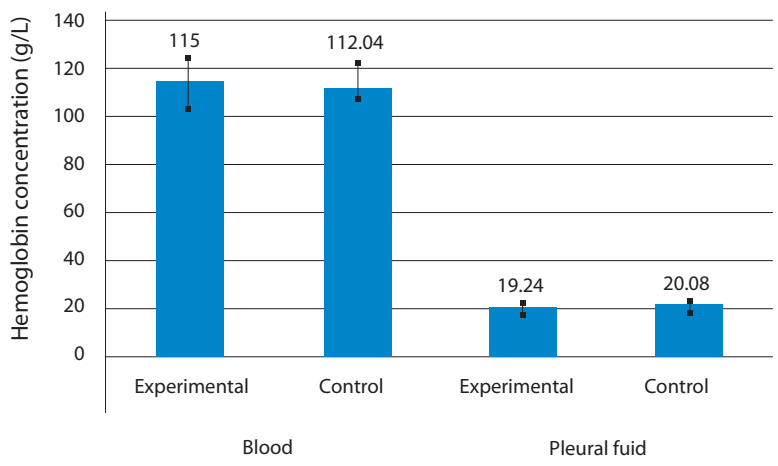

Fig. 3. Haemoglobin concentration in blood and pleural fluid on the first postoperative day. Values are expressed as the mean hemoglobin concentration $(\mathrm{g} / \mathrm{L}) \pm \mathrm{SE}$

Injuries of major anatomical structures as pulmonary vessels, bronchi, trachea and pericardium due to blood sample collection were not observed. Minor hemorrhage from pulmonary vein needle puncture site was observed in 5 patients $(20 \%)$. Duration of bleeding was restricted to several minutes (ranged $1-5$ minutes) and related to the time necessary for pulmonary vein ligation.

There was no difference in hospital stay duration ( $7 \pm 2$ days in experimental group versus $8 \pm 3$ days in control group, $\mathrm{p}>0.05)$. Chest tube drainage was longer in experimental group ( $6 \pm 4$ days) than in control group $(5 \pm 2), p=0.04$ (Fig. 4) 


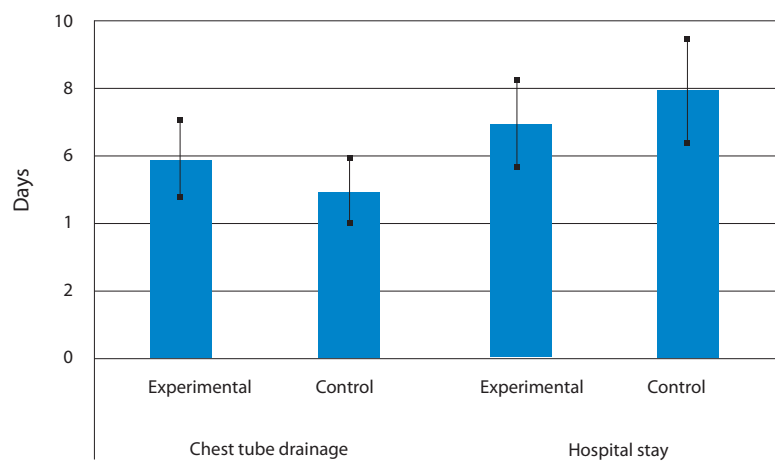

Fig. 4. Hospital stay and chest drainage duration. Values are expressed as the mean number of days \pm SE

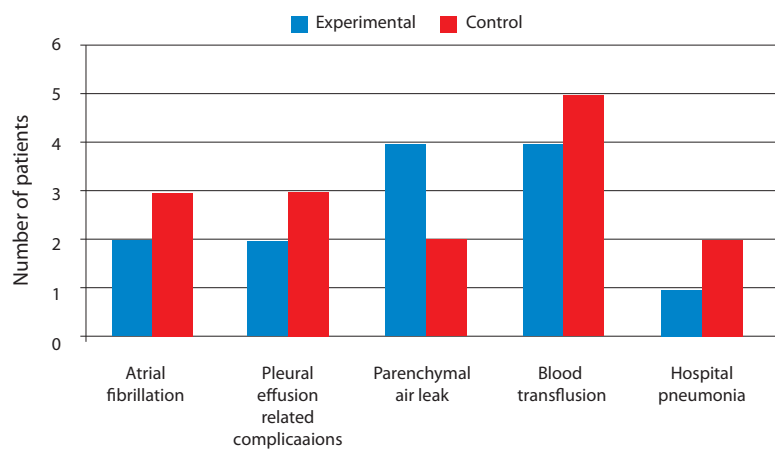

Fig. 5. Postoperative morbidity

There were no perioperative deaths. Cardiac complications were noted in one of the cases in experimental group (4\%) and three of the cases in control group (12\%) - atrial fibrillation developed and required treatment. In all cases arrhythmia successfully resolved during hospital stay. Four patients from experimental group (16\%) and five patients from control group $(20 \%)$ were given blood transfusion.

In two experimental cases (8\%) and three control cases (12\%) effusion-related complications developed that necessitated antibiotic treatment and prolonged drainage. Four patients from experimental group (16 $\%)$ and three patients from control group (12\%) had parenchymal air leaks persisting for more than 7 days. There were two cases of hospital pneumonia in control group (8\%) (Fig. 5.).

\section{DISCUSSION}

Research in biomarker field and discovery of novel potential biomarkers of NSCLC is essential and important as it is promising strategy to reduce lung cancer mortality. Single peripheral blood sample or in vitro tumor models not always adequately reflect complex metabolic processes and cellular interaction which can be found in vivo. Molecular theories based on experimental findings in vitro have limited impact on progress in cancer research $(12,15)$. On the other hand many clinical studies fail to isolate particular pathways in cancer cell biology due to complexity of molecular mechanisms, versatile function of receptors and ligands, as well as individual nature of every tumor based on unique genetical information. Up to date there is limited range of methods which make detailed analysis of cancer biology in vivo possible (2). As a rule with few exceptions these methods are invasive and ethical issues narrow spectrum of methods to be applied in human in clinical setting. Applied method allowed us to collect valuable material for further analysis with no impact on morbidity and minimal impact on course of surgical procedure.

Presence of pleural adhesions can sufficiently prolong the time of procedure, but what is more important excessive tissue trauma during mobilization of lung can cause metabolic changes and affect potential lung cancer biomarker profile $(3,18)$. One of the main goals during surgery was careful handling of lung tissue, maximally short time interval between skin incision and collecting blood samples to minimize systemic response to general anesthesia and surgical trauma.

Variations of pleural fluid drainage can reflect extent of surgical trauma $(4,6,11)$. Chest tube drainage duration was longer in experimental group, but further analysis revealed prevalence of lower lobectomies in experimental group. It is known, that due to anatomical features absorption of pleural fluid is more impaired in case of lower lobectomy than middle or upper lobectomy $(8,13)$.

Careful handling of pulmonary vessels during surgery is essential $(10,17)$, because minor tear in blood vessel wall can change course of operation or even affect extent of pulmonary resection. Major risk associated with novel approach is bleeding and injury of hilar structures which did not occur in any of 25 experimental cases. According to our results cardiac complications were not related to experimental procedure and occurred more often in control group, indicating possible relation to surgery itself.

One of main issues is use of analytic methods that do not provide precise and accurate determination of potential tumor specific proteins that are expressed in very low concentrations resulting in false negative results (1) gaining access to tumor microcirculation and its unique environment holds promise to solve this problem.

\section{CONCLUSIONS}

Combination of surgical practice with new research strategies can help to achieve progress in NSCLC biomarker discovery. Pulmonary resection remains a procedure containing a high risk of postoperative complications by itself, but application of new method for developing multimodal lung cancer model is safe and does not increase the risk of intraoperative and postoperative complications. 
Conflict of interest: None

\section{REFERENCES}

1. Aoki T, Tsuchida M, Watanabe T, Hashimoto T, Koike T, Hirono T, Hayashi J. Surgical strategy for clinical stage I non-small cell lung cancer in octogenarians. // Eur J Cardiothorac Surg, 2003; 23(4):446-450.

2. Balgkouranidou I, Liloglou T, Lianidou ES. Lung cancer epigenetics: emerging biomarkers. // Biomark Med, 2013; 7(1):49-58.

3. Chida M, Minowa M, Karube Y, Eba S, Okada Y, Miyoshi S, Kondo T. Worsened long-term outcomes and postoperative complications in octogenarians with lung cancer following mediastinal lymphnode dissection. // Interact CardioVasc Thorac Surg, 2009; 8(1):89-92.

4. Das-Neves-Pereira JC, Bagan P, Coimbra-Israel AP, Grimaillof-Junior A, Cesar-Lopez G, Milanez-deCampos JR, Riquet MR, Biscegli-Jatene F. Fasttrack rehabilitation for lung cancer lobectomy: a five-year experience. // Eur J Cardiothorac Surg, 2009; 36(2):383-392.

5. Gao WM, Kuick R, Orchekowski RP, et al. Distinctive serum protein profiles involving abundant proteins in lung cancer patients based upon antibody microarray analysis. // BMC Cancer, 2005; 5:110.

6. Giovannetti R, Alifano M, Stefani A, Legras A, Grigoroiu M, Collet JY, Magdelenat P, Regnard JF. Surgical treatment of bronchiectasis: early and long-term results. // Interact CardioVasc Thorac Surg, 2008; 7(4):609-612.

7. Ferguson MK, Celauro AD, Vigneswaran WT. Validation of a modified scoring system for cardiovascular risk associated with major lung resection. // Eur J Cardiothorac Surg, 2012; 41(3):598-602.

8. Ferguson MK and Durkin AE. A comparison of three scoring systems for predicting complications after major lung resection. // Eur J Cardiothorac Surg, 2003; 23(1):35-42.

9. Khan N, Cromer CJ, Campa M, et al. Clinical utility of serum amyloid A and macrophage migration inhibitory factor as serum biomarkers for the detection of nonsmall cell lung carcinoma. // Cancer, 2004; 101:379-384.

10. Kojima F, Yamamoto K, Matsuoka K, Ueda M, Hamada H, Imanishi N, Miyamoto Y. Factors affecting survival after lobectomy with pulmonary artery resection for primary lung cancer. // Eur J Cardiothorac Surg, 2011; 40(1):13-20.

11. Kouritas VK, Zissis C and Bellenis I. Variation of the postoperative fluid drainage according to the type of lobectomy. // Interact CardioVasc Thorac Surg, 2013; 16(4):437-440.
12. López E, Cho WC. Phosphoproteomics and lung cancer research. // Int J Mol Sci, 2012; 13(10):12287-314.

13. Matsuoka H, Okada M, Sakamoto T, Tsubota N. Complications and outcomes after pulmonary resection for cancer in patients 80 to 89 years of age. // Eur J Cardiothorac Surg, 2005; 28(3):380383.

14. Paesmans M. Prognostic and predictive factors for lung cancer. Review. // Eur Respir J Breathe, 2012; 9:113-122.

15. Pastor MD, Nogal A, Molina-Pinelo S, Meléndez R, Romero-Romero B, Mediano MD, López-Campos JL, García-Carbonero R, Sanchez-Gastaldo A, Carnero A, Paz-Ares L. Identification of oxidative stress related proteins as biomarkers for lung cancer and chronic obstructive pulmonary disease in bronchoalveolar lavage. // Int J Mol Sci, 2013; 14(2):3440-55.

16. Patz EF Jr, Campa MJ, Gottlin EB, et al. Panel of serum biomarkers for the diagnosis of lung cancer. // J Clin Oncol, 2007; 25:5578-5583.

17. Sakuragi T, Sakao Y, Furukawa K, Rikitake K, Ohtsubo S, Okazaki Y, Natsuaki M, Itoh T. Successful management of acute pulmonary embolism after surgery for lung cancer. // Eur J Cardiothorac Surg, 2003; 24(4):580-587.

18. Szczesny TJ, Slotwinski R, Stankiewicz A, Szczygiel B, Zaleska M, Kopacz M. Interleukin 6 and interleukin 1 receptor antagonist as early markers of complications after lung cancer surgery. // Eur J Cardiothorac Surg, 2007; 31 (4):719-724.

19. Taenzer A, Alix-Panabières C, Wikman H, Pantel K. Circulating tumor-d erived biomarkers in lung cancer. // J Thorac Dis, 2012; 4(5):448-9.

20. Wang Y, Hu Y, Wang D, Yu K, Wang L, Zou Y, Zhao C, Zhang $X$, Wang $P$, Ying $K$. The analysis of volatile organic compounds biomarkers for lung cancer in exhaled breath, tissues and cell lines. // Cancer Biomark, 2012; $11(4): 129-37$.

\section{Address:}

Artjoms Spaks

Thoracic Surgery Department,

Pauls Stradins Clinical University Hospital

Pilsonu 13, LV 1002, Riga, Latvia

E-mail: artjomsspaks@gmail.com 